\title{
Toxicity awareness and unintended suicide in drug overdoses
}

\author{
B. P. MCNICHOLL \\ Accident and Emergency Department, Royal Victoria Hospital, Belfast
}

\section{SUMMARY}

The objective of this paper is to determine patients awareness of the toxicity of the drugs they overdose with, the source of these drugs, and whether they would have taken them had they been fully aware of their toxicity, and to examine the implications for prevention.

A prospective review of one hundred consecutive overdoses admitted through an A\&E department was carried out. Awareness was scored by a ranking questionnarie and intent by response to a standard question when toxicity was explained.

Paracetamol was most the most frequently taken drug (39 cases). Overall awareness of toxicity was low. Twenty-eight patients $(30 \%)$ said they would not have overdosed if they had been aware of the toxicity of what they had taken. Fortynine per cent of patients took someone elses tablets.

There is a rise in analgesic poisoning from 1976. Higher toxicity awareness may reduce the incidence of drug overdose. Smaller prescriptions and over the counter preparations especially paracetamol and limited access to toxic drugs, especially in high-risk homes, should reduce both the number and severity of overdoses. It is possible that a number of deaths from deliberate drug overdose may be unintentional as $30 \%$ of survivors in this study did not intend the toxic effects of the drug taken.

\section{INTRODUCTION}

The problem of deliberate drug overdose continues since its peak in the 1970's with little impact being made by health professionals on the number of deaths (Meredith \& Vale, 1984) despite influencing the types of drugs taken (Meredith \&

Correspondence: B. P. McNicholl, Registrar, Accident and Emergency Department, Royal Victoria Hospital, Grosvenor Road, Belfast BT126BA. 
Vale, 1984; McMurray et al., 1985; Platt et al., 1988). The problem remains, being responsible for $4-5 \%$ of hospital discharges in the 15-44 age group (Forster, 1985), or 10\% of acute medical admission in 1985 (Kessel, 1985) and 798 deaths per annum in England and Wales and a further 530 deaths which may have been deliberate (OPCS, 1990). Paracetamol alone or in combination was the leading cause of drug-related deaths (559) in 1982 (England and Wales) (OPCS, 1988) and there has been a steady rise in the number of paracetamol and analgesic overdoses since then (McMurrary et al., 1985). A proportion of these may be preventable by reducing availability (Forster, 1985; McMurray et al., 1985; Platt et al., 1985), awareness of toxicity, (McMurray et al., 1985) primary prevention (Platt et al., 1985) but these can be difficult to realize (Bancroft et al., 1979; Platt et al., 1985).

Correlation between lethality of overdose, intent and outcome has been shown to vary in patients (Beck et al., 1975). The aim of this study was to pursue this further and ascertain how many patients would not have overdosed if their awareness of drug toxicity had been greater, and to discuss the implications for prevention.

\section{METHODS}

One hundred consecutive overdoses intending self harm admitted to the Royal Victoria Hospital through the Accident and Emergency unit were interviewed by the author. The interviews took place on the day after admission or later if toxic effects persisted. Psychotic and uncooperative patients or those whom the author felt were unreliable were not interviewed but objective data about them were obtained from other sources.

The A\&E department has a policy of admitting all overdoses and children under 13 years are not seen at the department. Only overdoses of drugs designed for human consumption were included (alcohol alone was excluded). Data collected included drugs ingested, where they were obtained and whether or not they were on prescription. Patients taking over the counter tablets were asked whether they had read the instructions on the packet and did they know the maximum recommended daily dose. Similar data were collected on the most recent previous overdose and whether or not the doctor precribing had taken measures to prevent recurrence. Patients were then assessed for toxicity awareness. Each patient was scored on a scale of $0-4$, as follows;

The patient was asked 'How harmful do you think an overdose of $\times$ is'? If the answer fitted one of the categories as listed below it was assigned that score, otherwise the patient was questioned as follows. Do you think this overdose would:

(0) Have no effect at all on you?

(1) Make you a little unwell?

(2) Make you sick enough to have to come into hospital?

(3) Make you extremely sick and close to dying?

(4) Kill you?

At the end of the interview patients were told the potential toxicity of their 
overdose in simple terms (in accordance with the U.K. Poisons Information Service toxicity data) as in the scale above, and asked 'If you had known that this overdose could have killed (or otherwise) harmed you would you have taken it?'. Answers were recorded as yes, no or don't know. Physical outcome was graded on a scale of 0-4, death scored four, end organ damage/dysrhythmias/convulsions scored 3, derangement of vital signs and or coma scale $<9$ scored 2, symptoms and coma scale $>8$ scored 1 , no physical effects scored 0 . The Kruskal-Wallis analysis of variance was used for statistical analysis.

\section{RESULTS}

Ninety-five patients fulfilled the inclusion criteria. Two patients were psychotic $\stackrel{0}{3}$ and three were unreliable (one violent, one uncooperative and one inconsistent in his replies) and only their objective data was included. Nine patients signed themselves out and 18 were discharged before being seen during the study and were not included. One patient who did not speak English and one who died in A\&E (prothiaden overdose) were excluded.

\section{Drugs taken}

Paracetamol was the most frequently taken tablet (38), 19 took it alone. Nineteen of the paracetamol overdoses took non-prescription tablets. Forty-five patients too alcohol with the overdose, 31 took non-barbiturate hypnotics, 11 NSAIDs (fou took aspirin), seven took tranquillizers, miscellaneous others, 29. Fifty-one patients took their own tablets, 49 took someone elses.

\section{Prescription status}

Only 26 overdoses contained non-prescription drugs, 7 of these were mixed with prescribed tablets. In three cases the prescription status was not known. Nine took their own non-prescription medication, four of these had read the instructions on the packet and only one knew the maximum recommended daily dose.

\section{Previous overdoses}

Thirty-three patients had taken a previous overdose. Sixteen of them took their $\frac{D}{9}$ own tablets on this occasion, and in 10 their doctor had taken measures to prevent another overdose.

\section{Awareness}

All patients (except one vitamin overdose) took a drug combination containing a potentially lethal drug (although the dose may not have been sufficient to kill in some cases). Patient awareness was significantly lower in those who said 'no' to the question 'would you have taken these tablets if you had known they could 
kill/seriously harm you?' $(P=0.03)$. Outcome scores for these patients were 3 in one case, 2 in seven cases and 1 in 15 cases. Forty-four of all patients had an awareness score of 1 or less. There was no significant difference in awareness between teenagers and adults or between those taking their own or someone elses tablets.

\section{Intent}

Twenty-eight patients said 'no' to the question 'would you have taken these tablets if you had known they could have killed (or otherwise harmed) you', 49 patients said yes, 18 patients did not know, five unsuitable patients were not asked. There was no significant difference between teenagers and adults.

\section{Outcome}

Five patients scored 3, the rest had minor side-effects e.g. drowsiness, or mild derangement of vital signs not requiring treatment. All were discharged physically well.

\section{DISCUSSION}

Forty-nine per cent of patients in this study took analgesics compared to $17.9 \%$ of overdoses in this hospital in 1976 (Hadden, 1978) in keeping with the rise in analgesic poisoning (McMurray et al., 1985). Less patients took antidepressants (4\% compared to $7.7 \%$ in 1976), there was also a drop in the number of nonbarbiturate hypnotics taken (31\% vs. $49 \%$ in 1976 , and $56.2 \%$ in 1975$)$ and no change in aspirin ingestion (4\%).

Many overdoses have low suicidal intent (Hawton et al., 1982) and take potentially lethal amounts (Beck et al., 1975). Although the reliability of patient response shortly after an overdose is questionable and therefore a methodological problem, it is an insoluble one because it relies on a subjective statement about an emotional issue and there is no gold standard by which to measure. Some of the patients in this study said they would not have overdosed if they were fully aware of toxicity. The real figure may be more or less than 28 patients but it is likely that some of these statements are true given the known discrepancy betwen professed and actual suicidal intent (Beck et al., 1975). The reference to 'unintended suicides' in the title refers to those persons who die of an overdose without meaning to kill themselves - a suicide gesture that becomes fatal. It is difficult to prove these deaths occur because intent is difficult to estimate in dying patients for various reasons but the low toxicity awareness in this study suggests that a number of these tragic cases exist; if there are surviors of unintended toxic overdoses there must be a number of deaths.

That a number of these patients are young with no underlying psychiatric illness and have good prognoses but overdose impulsively for trivial reasons 
(Bancroft et al., 1979) makes a pressing case for prevention. Awareness programs have not been implemented in any comprehensive way in the adult populations but in this study $30 \%$ of parasuicides might have been prevented through higher awareness of toxicity.

Based on the results there are two methods suggested to prevent or minimize the toxicity of overdoses.

(1) Reduce the number of tablets available for overdosage at any one time by restricting the size of prescription and over the counter supplies for all patients, when it is not inconvenient to do so, (as half the patients in this study took someone elses tablets) especially those at risk or living with a high-risk patient. Decreased substance availability is linked with less overdoses (Young, 1980; Forster, 1985; McMurray et al., 1985; Platt et al., 1988). The need to limit the access of repeat overdose patients to all medication must be reiterated.

(2) Promote toxicity awareness. Over the counter paracetamol packages for example do not warn of the possibly fatal effects of exceeding the maximum daily dose.

It is well known that the 'the principal opportunity for suicide prevention is in the physicians office' (Murphy, 1969). This also applies to parasuicide (Crockett, 1987 ) in the form of primary prevention, but as shown here promoting toxicity awareness may also prevent overdoses. Smaller prescriptions, especially of paracetamol, would do much to reduce overdose toxicity and encourage patients to limit the access of others, especially those at risk, to drugs in homes would also aid prevention. The number of 'unintended suicides' is not known. The findings in this and other studies (Beck et al., 1979) suggest a number of these cases existo An estimate of the number of unintended deaths due to self-poisoning is needede to determine the extent of the problem.

\section{CONCLUSIONS}

The rise in analgesic self-poisoning continues. Awareness of the toxicity of analgesics and other drugs taken is low. Thirty per cent of patients said they would not have overdosed if they had been aware of the toxicity of what they had taken. It is likely that a proportion of patients who die of an overdose do so unintentionally as $30 \%$ of survivors overdosed unintentionally in this study. Higher toxicity awareness and reduction of the volumes of toxic tablets available should reduce the number of 'unintended suicides' and the severity of parasuicides. An estimate of the incidence of 'unintended suicides' is needed.

\section{ACKNOWLEDGEMENTS}

Dr C. Patterson for his statistical advice and the late Dr P. Nelson and S/N H Fleming for their assistance. 


\section{REFERENCES}

Annual Report OPCS (1990) Mortality Statistics, pp. 88-90. HMSO, London.

Bancroft J., Hawton K., Simkin S., Kingston B., Cummings C. \& Whitwell D. (1979) The reasons people give for taking overdoses: a further inquiry. Journal of Medical Psychology 52, 353-365.

Beck A. T., Beck R. \& Kovacs M. (1975) Classification of suicidal behaviours: Quantifying intent and medical lethality. American Journal of Psychiatry 132(3), 285-287.

Crockett A. W. B. (1987) Patterns of consultation and parasuicide. British Medical Journal 295, 476-478.

Forster D. P. \& Frost D. E. B. (1985) Medicinal self-poisoning and prescription frequency. Acta Psychiatrica Scandinavica 71, 567-574.

Hadden D. S. M. (1978) Drug overdosage: a study at the Accident and Emergency Department of the Royal Victoria Hospital, Belfast. Ulster Medical Journal 47(2), 165-170.

Hawton K., Cole D., O'Grady J. \& Osborn M. (1982) Motivational aspects of deliberate self-poisoning in adolescents. British Journal Psychiatry 141, 286-291.

Kessel N. (1985) Patients who take overdoses. British Medical Journal 290, 1297-1298.

McMurray J. J., Northridge D. B., Abernethy V. A. \& Lawson A. H. (1987) Trends in analgesic poisoning in West Fife 1971-1985. Quarterly Journal of Medicine New series 65(246), 835-843.

Meredith T. J. \& Vale J. A. (1984) Epidemiology of analgesic overdose in England and Wales. Human Toxicology 3, 61s-74s.

Murphy G. E. (1969) Recognition of suicidal risk: the Physicians responsibility. Southern Medical Journal, 62, 723-728.

Osselton M. D. et al. (1984) Poisoning-associated deaths for England and Wales between 1973 and 1980. Human Toxicology 3, 201-221.

Platt S., Hawton K., Kreitman N., Fagg J. \& Foster J. (1988) Recent clinical snd epidemiological trends in parasuicide in Edinburgh and Oxford a tale of two cities. Psychological Medicine 18, 405-418. 